\title{
Milliliter per Hour
}

National Cancer Institute

\section{Source}

National Cancer Institute. Milliliter per Hour. NCI Thesaurus. Code C66962.

A metric unit of volumetric flow rate defined as the rate at which one milliliter of matter crosses a given surface during the period of time equal to one hour. 